\title{
Tom Mannewitz
}

\section{»Eine wirklich schöne nagelneue Welt«}

\author{
Kohärenzdominiertes Denken und politischer Extremismus
}

\section{Einleitung}

Politischer Extremismus hat den demokratischen Verfassungsstaat zu dessen Entstehungszeit herausgefordert, er fordert ihn derzeit heraus und er wird ihn in Zukunft herausfordern. Einem normativen Verständnis zufolge kennzeichnet der Begriff politische Phänomene, »die sich in der Ablehnung des demokratischen Verfassungsstaates und seiner fundamentalen Werte und Spielregeln einig wissen $\ll .{ }^{1}$ Diese Definition stellt den gemeinsamen Nenner aller Extremismen dar, eine einheitliche Ideologie existiert derweil nicht. Wer einen Blick auf die Phänomene wirft, die sich als Feinde einer offenen Gesellschaft zu erkennen geben, der sieht allerdings weitere Gemeinsamkeiten: Was Extremisten jedweder Couleur verbindet, sind bestimmte Strukturmerkmale in ihren Doktrinen - Freund-Feind-Stereotypien und Absolutheitsansprüche etwa.

Dass sich derartige Strukturmerkmale bei Extremisten konzentrieren, dürfte kein $\mathrm{Zu}$ fall sein: Sie lassen sich von der Grundannahme von Extremisten ableiten, die Akteure, Prozesse und Strukturen der Politik stünden in von bestimmten Gesetzmäßigkeiten und daher von einer bestimmten Logik determinierten Verhältnissen zueinander; davon unabhängige, zufällige, unintendierte und überraschende Phänomene gebe es in der Politik nicht. Dieser nachfolgend als Kohärenzprinzip bezeichneten Prämisse ordnen Extremisten alles Weitere unter. Ich werde zeigen, wodurch es sich auszeichnet und wie sich anhand dieses Prinzips die Feindschaft von der Zuneigung gegenüber der offenen Gesellschaft abgrenzen lässt. Anschließend sind die Strukturmerkmale des Extremismus ${ }^{2}$ vom Kohärenzprinzip zu deduzieren. Die Ursachen stehen anschließend im Vordergrund: Vieles deutet auf das unter Extremisten verbreitete, sozialpsychologische Phänomen der Ambiguitätsintoleranz, das es ihnen nicht erlaubt, »die Heterogenität und Vieldeutigkeit der Welt, die Kompliziertheit der Lebensverhältnisse und die Konflikthaftigkeit der Gesellschaft als Tatsachen anzuerkennen und konstruktiv umzusetzen «. ${ }^{3}$

1 Uwe Backes / Eckhard Jesse, Politischer Extremismus in der Bundesrepublik Deutschland, Berlin 1993, S. 40.

2 Vgl. Armin Pfahl-Traughber, »Gemeinsamkeiten im Denken der Feinde einer offenen Gesellschaft. Strukturmerkmale extremistischer Ideologien « in: ders. (Hg.), Jabrbuch Extremismusund Terrorismusforschung 2009/2010, Brühl 2010, S. 9-32.

3 Uwe Backes, »Extremismus: Konzeptionen, Definitionsprobleme und Kritik « in: ders. / Eckhard Jesse / Alexander Gallus (Hg.), Jahrbuch Extremismus E Demokratie. Bd. 22, Baden-Baden 2010, S. 25. 
Die Ableitung extremistischer Denkstrukturen von kohärenzbestimmtem Denken soll helfen, den Ursprung der Feindschaft gegenüber dem demokratischen Verfassungsstaat zu verstehen. Wer Menschen ins Lager der Befürworter einer offenen Gesellschaft holen will, muss ihre Denkweisen verstehen lernen, sich fragen, »wie Extremisten ticken $\ll$.

Der Extremismusbegriff ist nachfolgend als kurzes Synonym für die allgemeine Feindschaft gegenüber der offenen Gesellschaft zu verstehen, die ihrerseits nicht nur die Ablehnung der tragenden Säulen des demokratischen Verfassungsstaates (Gewaltenkontrolle, Pluralismus und Menschenrechte) impliziert, sondern sich gegen einige weitere Prinzipien wendet: individuelle Verantwortlichkeit, vielfältiges Entwicklungspotential einer Gesellschaft oder das, was Karl Popper die »Sozialtechnik der Einzelprobleme « genannt hat: ein evolutionäres Verständnis von gesellschaftlicher Entwicklung.

\section{Elemente kobärenzdominierten Denkens}

Extremisten jedweder Couleur sind bestrebt, aus dem politischen Leben erwachsende kognitive Dissonanzen ${ }^{5}$ zu vermeiden (Siehe folgender Abschnitt). Sie weigern sich deshalb, Widersprüche zwischen politischer Realität und eigener Erwartung sowie Mehrdeutigkeiten und Zufälligkeiten in der Politik anzuerkennen - etwa, wenn ihr positives Bild von einem charismatischen Politiker mit als negativ wahrgenommenen Informationen konfrontiert wird, für eine Wirtschaftskrise höchst unterschiedlich bewertete Ursachen angeführt werden oder Politiker unbeabsichtigt folgenreiche Fehler begehen. Stattdessen wollen sie die politische Realität bestimmten, nachvollziehbaren Gesetzmäßigkeiten unterworfen wissen, welche die treffsichere Prognose politischer Entwicklungen erlauben. Für sie ergäbe sich damit eine logisch aufgebaute politische Welt, in der menschliche Fehler, Zufälle und Irrtümer nicht aufträten. »Kohärenz heißt [...] >System< und dieses ist durch zweierlei ausgezeichnet: durch Konsistenz (Widerspruchsfreiheit) und Umfassendheit (>comprehensiveness $<$ ) ${ }^{6}{ }^{6}$

Extremistische Ideologien greifen dieses Bedürfnis auf, indem sie behaupten, die politische Realität sei, erstens, nur durch eine - ihre - Ideologie eins zu eins abbildbar, und, zweitens, sie sei genauso, wie genannte Menschen sie sich vorstellen: determiniert durch bestimmte Regeln und daher logisch aufgebaut. Der Kommunismus und der Nationalsozialismus etwa sind nach Lothar Fritze »darauf gerichtet, Politik aus anerkannten Gesetzmäßigkeiten abzuleiten. « ${ }^{7}$ Diese Gesetzmäßigkeiten fungieren als »Kleber « der politischen Realität und sorgen für einen kohärenten Aufbau der Politik. Der Marxismus etwa setzt die komplexen wirtschaftlichen Krisen mithilfe des historischen Materialismus

4 Karl R. Popper, Die offene Gesellschaft und ibre Feinde (1). Der Zauber Platons, München 1980, S. 214.

5 Siehe Leon Festinger, A Theory of Cognitive Dissonance, Stanford 1957.

6 Lourencino Bruno Puntel, Wabrheitstheorien in der neueren Philosophie, Darmstadt 1978, S. 173.

7 Lothar Fritze, Anatomie des totalitären Denkens. Kommunistische und nationalsozialistische Weltanschauung im Vergleich, München 2012, S. 67.

ZfP 60. Jg. 1/2013 
in einen klaren Bedeutungszusammenhang, der nationalsozialistische Sozialdarwinismus erklärt, dass Asylanten in jedem Fall eine Bedrohung des eigenen Wohlstands und der eigenen Identität seien - unabhängig davon, ob Fakten diese Aussage stützen. Die individualpsychologische Funktion der Kohärenzannahme ist Komplexitätsreduktion.

Wie ein Ertrinkender das Festland, so empfangen diese Menschen die Strukturiertheit, Widerspruchsfreiheit und Eindeutigkeit extremistischer Ideologien. Dafür müssen sie dem Axiom zustimmen können, nur eine bestimmte Ideologie könne die politische Wirklichkeit eins zu eins abbilden, während die durch Sinne erfahrbare Wirklichkeit allenfalls den Anschein der tatsächlichen Realität erwecke. Dies hat verschiedene Implikationen:

Wenn sie die Wahrheit einer Aussage über die soziale Wirklichkeit (empirische Aussagen $)^{8}$ überprüfen, machen Extremisten nichts anderes, als diese Aussage mit dem zu vergleichen, was sie für die Realität halten. Das unterscheidet sie nicht von Befürwortern einer offenen Gesellschaft. Allerdings: Extremisten überprüfen die Aussage nicht anhand der Empirie, sondern anhand der Weltanschauung, die sie für eine äquivalente Abbildung der Realität halten. Als empirisch wahr gilt ihnen nicht etwa, was mit der physisch erfahrbaren Realität korrespondiert, sondern was mit ihrer Ideologie logisch übereinstimmt. Dadurch schleicht sich das Kohärenzprinzip, das eigentlich logischen Aussagen ${ }^{9}$ vorbehalten ist, gleichsam durch die Hintertür in ihr Realitätsverständnis ein. Formal gesprochen, ersetzen sie - ohne es zu wollen - das bei empirischen Aussagen geltende Korrespondenzprinzip durch das Kohärenzprinzip. Damit ist nicht ausgeschlossen, dass ihre empirischen Aussagen mit der durch Sinne erfahrbaren Realität übereinstimmen können; nur ist dies nicht das Wahrheitskriterium. Dies zeigt sich bei denjenigen empirischen Aussagen, für die sie Wahrheit beanspruchen und bei denen die physische Wahrnehmung des empirischen Referenten ihre Aussage als empirisch falsch qualifiziert.

Wer versucht, mithilfe empirischer Beweise die Aussagen ihrer Ideologie der Unwahrheit zu überführen, stößt in aller Regel auf taube Ohren: Unbewusst führen Extremisten das Falsifizierbarkeitsprinzip ad absurdum, denn sie überprüfen den Wahrheitsgehalt ihrer auf die Realität bezogenen Aussagen nicht an der erfahrbaren Empirie, die Extremisten allenfalls für einen Anschein der Wirklichkeit halten, sondern an der eigenen Ideologie, die, weil sie die sozialen Gesetze des gesellschaftlichen Lebens formuliert, als »eigentliche« Realität betrachtet wird. Sie werten Aussagen als wahr, wenn sie »gemeinsam mit allen anderen verfügbaren Aussagen ein kohärentes Aussagengefüge bilden, wenn also die Aussagen einander nicht widersprechen $\ll{ }^{10}$ Paradoxerweise sind empiri-

8 Empirische Aussagen formulieren Behauptungen über bestimmte Ausschnitte der Realität, deren Gültigkeit sich durch Erfahrung bestimmen lässt, also durch das Einholen von Informationen über den empirischen Referenten. Um empirisch wahr zu sein, muss eine solche Aussage mit dem von ihr beschriebenen Wirklichkeitsausschnitt übereinstimmen (Korrespondenzprinzip).

9 Logische Aussagen formulieren logische Ableitungen innerhalb von bestimmten Denksystemen, deren Gültigkeit sich ohne Erfahrung, allein mit logischer Analyse nachweisen lässt. Das zur Geltung kommende Wahrheitskriterium bei logischer Wahrheit ist die Kohärenz gegenüber anderen logischen Aussagen desselben Denksystems (Kohärenzprinzip).

10 Werner J. Patzelt, Sozialwissenschaftliche Forschungslogik. Einführung, München 1986, S. 91. 
sche Aussagen von Extremisten also in erster Linie antiempirisch, weil logisch und deshalb - jedenfalls in ihren Augen - nicht durch Fakten zu widerlegen. Extremisten sind einer auf Tatsachen gestützten Beweisführung daher nicht zugänglich. Was bleibt, ist eine stimmige Weltsicht ohne Widersprüche und böse Überraschungen, allerdings zum Preis der Emanzipation von der realen Welt und einer eng umgrenzten Fähigkeit, Neues zu erfahren und durch Fehler zu lernen, denn Lernen setzt einen Widerspruch zwischen der eigenen Erwartungshaltung und der Erfahrung voraus.

Dieses Vorgehen ist Selbstschutz: Akzeptierte etwa ein Rechtsextremist geringe Immigrationszahlen als Beweis, dass Ausländer schon gar keine Bedrohung für die heimische Wirtschaft darstellen können, führte er seine als Deutungsrahmen fungierende, rechtsextremistische Ideologie ad absurdum: Sie wäre kein Deutungsrahmen mehr, sondern nur noch eine leere Hülle aus logisch miteinander verbundenen Aussagen. Deshalb stellen Extremisten die Falsifizierbarkeit (d.h. empirische Überprüfbarkeit) empirischer Aussagen infrage und immunisieren sich so gegen die Realität.

Wie wird jedoch bestimmt, welche von zwei sich widersprechenden Aussagen, die mit je einem Aussagensystem, je einer Ideologie kohärent sind, wahr ist? Die Frage kann nur mit Verweis auf die Wirklichkeit beantwortet werden. Damit bricht extremistisches Denken allerdings mit dem Prinzip, als Referenzrahmen nur die eigene Ideologie zu nutzen. Diese Inkonsistenz leistet der willkürlichen Auswahl von Tatsachen Vorschub, die das eigene Aussagensystem - etwa den Trotzkismus - stützen, das alternative System - zum Beispiel den Maoismus - aber als unwahr diskreditieren. Die Ideologie stützt sich »nur auf einen einzigen, noch der erfahrenen Wirklichkeit entnommenen Punkt, der in der Prämisse als gegeben angenommen wird, [...], [lässt] die von hier aus entfaltete Bewegung dann aber von aller weiteren Erfahrung völlig unberührt «. ${ }^{11}$ Den Boden des Antiempirismus verlässt der Extremismus folglich nur, wenn er die Kohärenz der eigenen Ideologie stützt; er ist damit willkürlich.

Wie auch der Kohärenztheorie der Wahrheit ${ }^{12}$ haftet dem kohärenzdominierten Denken ein antiempirisches Moment an, ist es doch prinzipiell möglich, dass wahre Aussagen über die Realität im Widerspruch zu ihr stehen, wenn sie sich nur in ein bereits bestehendes, vollständiges Aussagensystem einfügen. Widerspruchsfreiheit und logische Deduzierbarkeit werden zu »allein selig machenden « Wahrheitskriterien bei Aussagen über die Wirklichkeit erhoben.

Das beschriebene Muster - die kompromisslose Unterordnung jeder Argumentation, jeder Idee und jedes Beweises unter der Prämisse, die politische Welt sei in jedem Falle logisch aufgebaut, die daraus resultierende Anwendung des Kohärenzkriteriums bei empirischen Aussagen und der Antiempirismus - wird ferner als kohärenzdominiertes, -getriebenes oder -gesteuertes Denken bezeichnet.

11 Hannah Arendt, Elemente und Ursprünge totaler Herrschaft. Antisemitismus, Imperialismus, totale Herrschaft, München 1986, S. $964 \mathrm{f}$.

12 Siehe Puntel, Wabrheitstheorien in der neneren Philosophie, aaO. (FN 6), S. 172-204.

ZfP 60. Jg. 1/2013 


\section{Kohärenzdominiertes Denken und die Abgrenzung der Feinde von den Freunden einer offenen Gesellschaft}

Die conditio sine qua non dafür, dass ein kohärenzdominiert denkender Menschen überhaupt dem Extremismus anheimfallen kann, ist seine Weigerung, die Widersprüchlichkeit der politischen - nicht der privaten, nicht der sportlichen, nicht der religiösen - Realität anzuerkennen.

Was nun aber ist ein hinreichendes Unterscheidungskriterium zwischen den Denkstrukturen eines Extremisten und denen eines Demokraten? Die Geltung des Kohärenzprinzips (Widerspruchsfreiheit und Umfassendheit) innerhalb logischer Aussagensysteme - etwa bestimmter Werte- oder Moralsysteme - kann nicht herangezogen werden, um liberale Demokraten von Extremisten zu unterscheiden, wohl aber die Geltung des Kohärenzprinzips bei empirischen Aussagen:

Feinde wie Freunde einer offenen Gesellschaft können der Auffassung sein, die politische Welt sei durch bestimmte, global wirkende Gesetzmäßigkeiten determiniert. Was sie unterscheidet, ist die Kompromisslosigkeit, mit der sie sich an diese Vorstellung klammern. Der Prüfstein ist die Frage, ob die Übereinstimmung mit der empirischen (nicht der dafür gehaltenen) Wirklichkeit ohne Wenn und Aber als Wahrheitskriterium empirischer Aussagen anerkannt wird: Während Befürworter einer offenen Gesellschaft in aller Regel die Wahrheit einer empirischen Aussage an der Wirklichkeit messen und so nötigenfalls die Widersprüchlichkeit und Mehrdeutigkeit der politischen Realität hinzunehmen bereit sind, gilt einem Extremisten hauptsächlich das als wahr, was sich in die Gesetzmäßigkeiten seiner Ideologie einfügt - selbst wenn das bedeutet, dass es sich mit der empirischen Wirklichkeit nicht so verhält. Die bedingungslose Anwendung des Kohärenzprinzips bringt seine Weigerung zum Ausdruck, die Imperfektibilität der politischen Realität anzuerkennen. Gleichwohl gibt es zwischen Extremisten und Demokraten mit Blick auf die Geltung der Kohärenzprämisse ein weites Feld der Abstufungen: Nur wer mehrheitlich das Verhaltensmuster zeigt, Aussagen als wahr zu werten, die im Widerspruch zur empirisch erfahrbaren Realität stehen oder nicht durch sie gestützt werden, gehört eher ins Lager des Extremismus. Damit dominiert das Prinzip der Kohärenz gegenüber dem Bedürfnis nach Wirklichkeitserfahrung.

Ein Beispiel: Die Aussage »Nationalsozialismus und eine Demokratie modernen westlichen Zuschnitts vertragen sich nicht « ist eine logische Aussage, denn ihr Wahrheitsgehalt lässt sich ohne Wirklichkeitserfahrung, allein durch einen Blick auf die Prinzipien beider Systeme überprüfen. Ein Rechtsextremist wie ein Demokrat werden übereinstimmend die Wahrheit dieser Aussage bestätigen, denn sie legen als Wahrheitskriterium logische Kohärenz zugrunde. Bei der empirischen Aussage »Das Dritte Reich hat etwa sechs Millionen Juden ermordet « können sie zu unterschiedlichen Ergebnissen kommen: Ein der offenen Gesellschaft gegenüber aufgeschlossener Mensch wird unter der erdrückenden Last der empirischen Beweise die Aussage bestätigen. Sein Wahrheitskriterium ist die Korrespondenz der Aussage mit der Wirklichkeit, die Falsifizierbarkeit der Aussage ist seine Grundannahme. Ein Rechtsextremist hingegen geht vermutlich einen anderen Weg: Er wird behaupten, mit der Vorlage noch so vieler Fakten und Beweise könne 
man die Wahrheit der Aussage nicht feststellen. Damit negiert er ihre Falsifizierbarkeit. Stattdessen wird er die Unwahrheit der Aussage dadurch zu untermauern versuchen, dass er ein umfassendes und konsistentes Aussagenkonstrukt heranzieht: Die belastenden Dokumente etwa seien von Juden gefälscht, die Leichen auf Fotos stellten Deutsche dar, welche die eigentlichen Opfer seien, die Fälschung sei kaum aufzudecken, weil »das Judentum « die Fäden der Welt in der Hand halte, weswegen eine auf ihre Dokumente gestützte Argumentation ins Leere laufen müsse, und vieles mehr. Die einzelnen Thesen und Theoreme stützen sich gegenseitig. Was sie außer der Kohärenz innerhalb eines einzigen Aussagensystems eint, ist ihre allenfalls selektive Bezugnahme auf Fakten. Ein Rechtsextremist wird den Holocaust erst dann anerkennen, wenn er damit nicht die Kohärenz seiner Ideologie gefährdet, etwa wenn es sich um einen überzeugten Nationalsozialisten handelt. Extremisten greifen also auf den Falsifikationismus zurück, wenn er die eigene Ideologie untermauert, und lehnen ihn ab, wenn er die Widerspruchsfreiheit bedroht.

\section{Kohärenzdominiertes Denken als Prinzip extremistischer Denkstrukturen}

Feinde der offenen Gesellschaft folgen in der Regel unbewusst einem kohärenzdominierten Denkmuster, treffen sie Aussagen über die politische Realität. Sie tun dies vor allem, um der Mehrdeutigkeit und Widersprüchlichkeit Herr zu werden, die bei ihnen kognitive Dissonanzen verursachen. Ein kohärentes Denksystem kann sie auflösen, denn die Falsifizierbarkeit empirischer Aussagen wird untergraben. Die Sicht auf die Realität wird selektiv. Nachfolgend leite ich extremistische Denkstrukturen ${ }^{13}$ von kohärenzdominiertem Denken ab:

1) Exklusiver Erkenntnisanspruch: Feinde der offenen Gesellschaft kennzeichnet der Anspruch, allein der Erkenntnis gesellschaftlicher Entwicklungen fähig zu sein. Aus dieser Einsicht in »höheres Wissen « leitet sich ein Anspruch auf das Interpretationsmonopol gesellschaftlicher, politischer und wirtschaftlicher Zusammenhänge ab. Sie speist sich aus dem Glauben an axiomatische Setzungen. Anhängern anderer politischer Richtungen wird die Erkenntnisfähigkeit abgesprochen. Woher nehmen Extremisten dieses Selbstbewusstsein, andere Ansichten als falsch, die eigene als einzig richtig anzusehen? Sie lässt sich dann aufrechterhalten, wenn das eigene Aussagensystem sich im Kampf mit der Komplexität politischer Wirklichkeit bewährt hat. Andere Aussagen(systeme), die solche logischen Gesetzmäßigkeiten nicht umfassen oder keine befriedigenden Antworten zu geben vermögen, müssten demnach abgelehnt werden, das eigene Aussagensystem wird $\mathrm{zu}$ »höherem Wissen«. Der Zwang zu widerspruchsfeien und umfassenden Aussagensystemen leistet damit dem exklusiven Erkenntnisanspruch Vorschub.

2) Dogmatischer Absolutheitsanspruch: Ebenso die Behauptung von Extremisten, sie hätten absolut wahre und allgemeingültige gesellschaftliche Prinzipien entdeckt, weswegen andere Orientierungsmaßstäbe und Kritiken abzuweisen seien, findet ihren Ur-

13 Vgl. Pfahl-Traughber, »Gemeinsamkeiten im Denken der Feinde einer offenen Gesellschaft. Strukturmerkmale extremistischer Ideologien«, aaO. (FN 2), S. 9-32.

ZfP 60. Jg. 1/2013 
sprung in kohärenzdominiertem Denken. Schließlich lassen sich politische Phänomene logisch mittels weniger, einfacher Gesetze erklären. Dass daraus Dogmatismus erwächst, liegt an der Dringlichkeit, mit der sie Widersprüche abzuwehren und komplexe Zusammenhänge der Realität in einen übersichtlichen Erklärungsrahmen zu integrieren bestrebt sind. Das Insistieren auf Widerspruchsfreiheit und Einfachheit nährt eine gewisse Immunität gegenüber Kritik, welche das Korrespondenzkriterium ins Spiel bringen und damit erneut kognitive Dissonanzen hervorrufen könnte. Abgewiesen werden solche Einwürfe zumeist mit dem Gegenvorwurf der Irrationalität.

3) Essentialistisches Deutungsmonopol: Das Denken der Feinde der offenen Gesellschaft ist geprägt vom Anspruch, das » Wesen hinter den Dingen «, ${ }^{14}$ also bestimmte, nicht direkt beobachtbare Gesetzmäßigkeiten, erkannt zu haben. Es ist damit aus philosophischer Sicht idealistisch. Der Ursprung in kohärenzgetriebenem Denken wird an dieser Stelle besonders deutlich, denn die eigene Ideologie muss eine Realität objektiv abbilden. Die Widersprüche zwischen Ideologie und physisch wahrnehmbarer Empirie lassen den Schluss naheliegend erscheinen, die Ideologie beschreibe Gesetzmäßigkeiten, deren Auswirkungen nicht durch Sinne erfahrbar sind. Diese Gesetze an konkreten Auswirkungen zu ermitteln müsse der Empiriker dann natürlich scheitern. Allein mit Logik sei ihnen auf die Schliche zu kommen. Mit diesem Argument wird die Kohärenz der eigenen Ideologie vor unbequemen Informationen immunisiert. Die Ablehnung der Übereinstimmung einer Aussage mit dem empirischen Sachverhalt als Wahrheitskriterium ist der Inbegriff einer antiempirischen Weltsicht, deren Blick nicht auf die Empirie, sondern das eigene Theoriegebäude, die eigene Ideologie gerichtet ist.

4) Holistische Steuerungsabsichten: Extremisten leiten - jedenfalls idealtypisch - ihre ganzheitlichen, totalitären Projekte ab von dem »Anspruch, ganzheitliche Aussagen über die Beschaffenheit und Entwicklung von Gesellschaften formulieren zu können «. ${ }^{15}$ Dieser Anspruch folgt der Übersetzung des Kohärenzkriteriums in die politische Sphäre. Widerspruchsfreie und umfassende Einsichten in die politische Praxis umzusetzen, erscheint nur rechtens ${ }^{16}$ denn es kann wegen der Geltung nur eines Aussagensystems lediglich eine politische Ideologie korrekt sein - warum also die politische Gestaltung durch andere akzeptieren, wenn doch die Politik bekannt ist, die in »Übereinstimmung mit den sozialen Gesetzmäßigkeiten ${ }^{17}{ }^{17}$ steht?

In ihrer philosophischen Auseinandersetzung mit den Totalitarismen des 20. Jahrhunderts erkannte Hannah Arendt den Zusammenhang zwischen diesen holistischen Steuerungsabsichten und dem Antiempirismus, als sie erklärte: »Sind die Bewegungen erst einmal an die Macht gekommen, so beginnen sie, die Wirklichkeit im Sinne ihrer

14 Pfahl-Traughber, »Gemeinsamkeiten im Denken der Feinde einer offenen Gesellschaft. Strukturmerkmale extremistischer Ideologien«, aaO. (FN 2), S. 17.

15 Pfahl-Traughber, »Gemeinsamkeiten im Denken der Feinde einer offenen Gesellschaft. Strukturmerkmale extremistischer Ideologien", aaO. (FN 2), S. 19.

16 Vgl. Fritze, Anatomie des totalitären Denkens. Kommunistische und nationalsozialistische Weltanschaunng im Vergleich, aaO. (FN 7), S. 67.

17 Fritze, Anatomie des totalitären Denkens. Kommunistische und nationalsozialistische Weltanschaunng im Vergleich, aaO. (FN 7), S. 74. 
ideologischen Behauptungen zu verändern. [...] Dem, was faktisch geschieht, kommt ideologisches Denken dadurch bei, daß es aus einer als sicher angenommenen Prämisse nun mit absoluter Folgerichtigkeit - und das heißt natürlich mit einer Stimmigkeit, wie sie in der Wirklichkeit nie anzutreffen ist - alles weitere deduziert. « ${ }^{18}$ Die Beweisführung der Feinde der offenen Gesellschaft ist auf logische Wahrheit, Deduktion und Vollständigkeit ausgerichtet. Ziel ist es, eine widerspruchsfreie, erschöpfende Politik zu generieren, selbst wenn das impliziert, die Tatsachen den Aussagen anzupassen. So kann der letzte Widerspruch - der zwischen Ideologie und Realität - aufgehoben werden.

5) Deterministisches Geschichtsbild: Den Feinden einer offenen Gesellschaft zufolge verläuft die geschichtliche Entwicklung nach einer bestimmten unveränderbaren Logik (Historizismus). Der Versuch der Einflussnahme auf diese Entwicklung durch den Menschen ist also sinn- und hoffnungslos. Diese Annahme beruht auf dem Anspruch, erkannt zu haben, dass es logische »soziale« Gesetze gibt, denn: Historizismus ist nichts anderes als eine Präzisierung der Annahme der Kohärenz der Wirklichkeit im Sinne einer festen Logik, die den Lauf der Dinge bestimmt. Die Geschichte entwickle sich nicht sprunghaft und unvorhersehbar weiter - so die Aussage. Vielmehr bewege sie sich in bestimmten Linien fort, weil sie einem Prinzip folge, das die - selektiv herangezogenen - Daten der Geschichte stimmig ins Verhältnis zueinander setze. Zwei historische Ereignisse verhielten sich zueinander folglich immer konsistent, da beide Folge ein und desselben Entwicklungsgesetzes seien. Widersprüchliche oder unerwartete Ereignisse: unvorstellbar.

6) Identitäre Gesellschaftskonzeption: Wie auch bei den holistischen Steuerungsabsichten geht es bei der identitären Gesellschaftskonzeption nicht um die Beschreibung der Welt, wie sie ist, sondern um die Welt, wie sie sein soll bzw. sein wird: Die prinzipielle Ablehnung von Aussagen, die mit dem eigenen Aussagensystem nicht konform gehen, indizieren die »Fixierung auf Einheit und Geschlossenheit, die in abweichenden Auffassungen oder konkurrierenden Interessen eine Gefahr für den Erhalt der Homogenität sieht.$^{19}$ Wenn es einen einheitlichen Volkswillen und politische Homogenität schon nicht in der Realität gibt, so müssen sie zumindest angestrebt werden. Nur so lässt sich ein ganzheitlicher, kohärenter Gesellschaftsansatz umsetzen, nur so kann sich das eigene - kohärente - Aussagensystem verbreiten, ohne Gefahr zu laufen, auf Widerstände zu stoßen.

7) Dualistischer Rigorismus: Das Denken der Feinde der offenen Gesellschaft in Polarisierungskategorien und Gegensatzpaaren (Freund - Feind, Gut - Böse, Sieger - Besiegter) entspringt zwei Prinzipien: Einesteils schafft es innerhalb eines extremistischen Aussagensystems klare Fronten: Ein- und demselben Element der Wirklichkeit - etwa einer bestimmten Person - kommt nur eine einzige, gesellschaftlich bedeutsame Rolle zu. In schwarzen Humor kleidet folgende, aus Nordirland stammende Anekdote jenes dualistische Denken: Auf die Frage zweier sich im Streit befindlicher Nordiren, ob er

18 Arendt, Elemente und Ursprünge totaler Herrschaft. Antisemitismus, Imperialismus, totale Herrschaft, aaO. (FN 11), S. 965. Kursivsetzung von der Autorin vorgenommen.

19 Pfahl-Traughber, »Gemeinsamkeiten im Denken der Feinde einer offenen Gesellschaft. Strukturmerkmale extremistischer Ideologien«, aaO. (FN 2), S. 23.

ZfP 60. Jg. 1/2013 
Katholik oder Protestant sei, antwortet der Ortsfremde, er sei Muslim. Verdutzt sehen sich die beiden Kontrahenten an und erwidern: »Schon klar, aber: ein katholischer Muslim oder ein protestantischer Muslim? «Anderenteils sorgen dualistische Rigorismen dafür, dass Aussagen, die dem eigenen System widersprechen, dadurch aufgehoben werden, dass diejenigen, die sie treffen, bekämpft und in letzter Konsequenz ausgelöscht werden.

8) Fundamentale Verwerfung: Die prinzipielle Ablehnung eines bestehenden demokratischen Verfassungsstaates durch seine Feinde kommt einer fundamentalen Verwerfung gleich. Um deren Charakter offen zu legen, muss danach gefragt werden, welchem Ziel das Bestehende geopfert werden soll. Es ist keineswegs ein nur in einigen Punkten verbesserter demokratischer Verfassungsstaat - etwa mit mehr sozialer Gleichheit, mehr Nationalstolz oder Beachtung der islamischen Religion. Die Feinde der offenen Gesellschaft wollen die Mängel der Demokratie radikal auslöschen. Es ist das, was Armin PfahlTraughber ein »idealisierte[s] Gesellschaftsmodell ${ }^{20}$ nennt und dem Karl Popper den Begriff des radikalen Ästhetizismus aufprägte: ${ }^{21}$ Der Kritische Rationalist sah bei Platon und Marx - dies gilt allerdings in idealtypischer Form für alle Feinde der offenen Gesellschaft - den »Wunsch, eine Welt zu bauen, die nicht nur ein wenig besser und vernünftiger ist als die unsrige, sondern die von all ihrer Häßlichkeit frei ist: Nicht einen aus alten Flecken zusammengesetzten Läufer will er [der extreme Radikalismus], sondern einen großen, ganz neuen Teppich, eine wirklich schöne nagelneue Welt. «22 Wie die holistischen Steuerungsabsichten und die idenitäre Gesellschaftskonzeption, so beschreibt die völlige Auslöschung des bestehenden Gesellschaftssystems zugunsten einer Utopie den zweiten Schritt. Der erste Schritt besteht darin, Widersprüche durch die Emanzipation von Realität und die Fokussierung auf ein einziges, widerspruchsfreies Aussagensystem zu vermeiden. Erst in einem zweiten Schritt - bei Herrschaftsantritt - wird die Realität dem logisch passenden Aussagensystem angepasst; nicht schrittweise, weil so widersprüchliche Elemente Bestand haben könnten, sondern durch die Installation eines gänzlich neuen, umfassenden Gesellschaftssystems, das die Gefahr überdauernder und daher potentiell widersprüchlicher Institutionen, Werte und Akteure bannt. Das Postulat der in sich stimmigen Wahrheit wird so zum politisch-gesellschaftlichen Traum.

Extremismus gibt der logischen Wahrheit vor der empirischen Wahrheit den Vorzug, um darüber zu entscheiden, ob eine Aussage über die Realität stimmt, und akzeptiert Widersprüche zur als irrelevant abgestempelten, erfahrbaren Wirklichkeit. Die ideologische Beweisführung sei, so Hannah Arendt, immer logisch deduzierend und unabhängig von menschlicher Erfahrung. ${ }^{23}$ Damit argumentiert ein idealtypischer Extremist in erster Linie antiempirisch. Er unterschlägt die Existenz von Widersprüchen, Zufällen

20 Pfahl-Traughber, »Gemeinsamkeiten im Denken der Feinde einer offenen Gesellschaft. Strukturmerkmale extremistischer Ideologien «, aaO. (FN 2), S. 27.

21 Vgl. Popper, Die offene Gesellschaft und ibre Feinde (1). Der Zauber Platons, aaO. (FN 4), S. 223.

22 Popper, Die offene Gesellschaft und ihre Feinde (1). Der Zauber Platons, aaO. (FN 4), S. 223.

$23 \mathrm{Vgl}$. Arendt, Elemente und Ursprünge totaler Herrschaft. Antisemitismus, Imperialismus, totale Herrschaft, aaO. (FN 11), S. $964 \mathrm{f}$. 
und Mehrdeutigkeiten in der Realität und ist - wenn er an die Macht gelangt - bestrebt, jene realen Widersprüche aufzuheben, »weil nicht sein kann, was nicht sein darf«.

\section{Ambiguitätsintoleranz}

Mit Ambiguitätstoleranz werden meist sehr ähnliche Phänomene bezeichnet, die Definitionen unterscheiden sich meist nur durch Nuancen. ${ }^{24}$ Eine umfassende Begriffsklärung stammt von Robert Norton: »Intolerance of ambiguity is a tendency to perceive or interpret information marked by vague, incomplete, fragmented, multiple, probable, unstructured, uncertain, inconsistent, contrary, contradictory, or unclear meanings as actual or potential of psychological discomfort or threat." ${ }^{25}$ Ambiguitätsintolerante Menschen reagierten laut Else Frenkel-Brunswik, der Begründerin dieses hypothetischen Persönlichkeitskonstruktes, ${ }^{26}$ auf derartige Reize »with a tendency to resort to black-white solutions, to arrive at premature closure as to valuative aspects, often at the neglect of reality, and to seek for unqualified and unambiguous over-all acceptance and rejection of other people. The maintenance of such solutions requires the shutting out of aspects of reality which represent a possible threat to these solutions." 27 Sie scheint ohne es geahnt zu haben - jenes kohärenzdominierte Denkmuster benannt zu haben, das für Feinde der offenen Gesellschaft typisch ist.

Ambiguitätstoleranz ist kein festes Persönlichkeitsmerkmal, sondern ein inhaltsspezifisches »Regulativ der Aufnahme-, Verarbeitungs- und Speicherungsprozesse $«{ }^{28} .{ }^{29}$ Aus ambiguitätsintolerantem Verhalten von Menschen gegenüber einem bestimmten Lebensaspekt (z. B. den Eltern) kann also nicht auf Ambiguitätsintoleranz gegenüber einem anderen Komplex (z. B. Politik) geschlossen werden. Gleichwohl indiziert die volle Vereinnahmung bzw. die prinzipiell ablehnende Haltung gegenüber einem Wirklichkeitsausschnitt, dass der betroffene Mensch diesen Ausschnitt als mehrdeutig wahrnimmt. Für ihn überschneiden sich positive wie negative, angenehme und unangenehme Aspekte.

Dass die Autoren um Adorno, allen voran Frenkel-Brunswik, bei ambiguitätsintoleranten Menschen vor allem Rechtsextremisten, in ihren Worten: Faschisten, im Blick

24 Vgl. für den Zusammenhang zwischen kognitiver Dissonanz und Ambiguitätstoleranz Siegfried Schumann, Persönlichkeitsbedingte Einstellungen zu Parteien, Oldenbourg 2001, S. 79-81.

25 Robert W. Norton, zitiert nach Jack Reis, Ambiguitätstoleranz. Beiträge zur Entwicklung eines Persönlichkeitskonstruktes, Heidelberg 1997, S. 7.

26 Vgl. Reis, Ambiguitätstoleranz. Beiträge zur Entwicklung eines Persönlichkeitskonstruktes, aaO. (FN 25), S. $7 \mathrm{f}$.

27 Frenkel-Brunswik arbeitete innerhalb der Berkeley-Gruppe bei der Studie zur »Authoritarian Personality « am Konzept der Ambiguitätstoleranz. Else Frenkel-Brunswik, »Intolerance of Ambiguity as an Emotional and Perceptual Personality Variable« in: Journal of Personality (18), S. 108-143, hier: 115.

28 Reis, Ambiguitätstoleranz. Beiträge zur Entwicklung eines Persönlichkeitskonstruktes, aaO. (FN 25), S. 9.

29 Vgl. Kevin Durrheim / Don Foster, »Tolerance of Ambiguity as a Content Specific Construct « in: Personality and Individual Differences (22), No. 5, S. 741-50.

ZfP 60. Jg. 1/2013 
hatten, war dem Eindruck des deutschen Nationalsozialismus und des italienischen $\mathrm{Fa}$ schismus geschuldet. Gleichwohl ist die Fixierung auf ambiguitätsintolerante Verhaltensmuster bei Menschen mit rechtsextremistischen Orientierungen nicht gerechtfertigt, wie in mehreren Arbeiten gezeigt werden konnte. ${ }^{30}$ Die oben genannten Denkstrukturen lassen sich also auf einen kognitiven Verarbeitungsstil von politischen Stimuli zurückführen, der durch Polarisierungskategorien, durch manichäische Denkstereotypen und radikale Lösungsvorschläge geprägt ist. Damit wird aus Sicht der Betroffenen wieder Klarheit und Sicherheit in eine Situation gebracht, die ihnen Unbehagen bereitet. Sie halten die Spannungen, die Vieldeutigkeit der Politik nicht aus. Mehrdeutige Reize scheinen demnach dann ambiguitätsintolerante Verarbeitungsstrategien (völlige Ablehnung oder totaler Zuspruch, Realitätsausblendung, radikale Lösungsvorschläge) zu provozieren, wenn Menschen diesem Bereich ambiguitätssensibel gegenüberstehen.

\section{Kohärenzdominiertes Denken als Verarbeitungsstrategie ambiguitätsintoleranter Menschen}

Kohärenzdominiertes Denken entwickelt sich dort zu Extremismus, wo Menschen ambiguitätsintolerant gegenüber der politischen Sphäre sind. Ambiguitätsintoleranz und kohärenzdominiertes Denken greifen dann ineinander wie Zahnräder, um die Fährnisse der politischen Realität zu bannen. Der zentrale Grund, der für die beiden Wahrheitskriterien Konsistenz und Umfassendheit spricht, ist deren Abwehr von kognitiver Dissonanz. Wer die Kohärenz einer Aussage mit dem eigenen Aussagen- oder Denksystem (das die Realität objektiv abbilden soll) als Maßstab für Wahrheit zugrunde legt, umgeht böse Überraschungen, die durch Widersprüche zwischen Erwartung und Realität entstehen können. Die empirische Beweislast kann noch so erdrückend sein - die Wahrheit lässt sich jemandem, der den Wahrheitsgehalt empirischer Aussagen an der Übereinstimmung mit den Gesetzmäßigkeiten des eigenen Aussagensystems misst, nicht durch Verweis auf die Wirklichkeit näherbringen. Er gibt damit ein gutes Stück Lernfähigkeit preis. »Die wirklich fanatischen Ewiggestrigen sind nicht empfänglich für Beweise. Sie glauben das, was sie glauben wollen mit der hermetischen Verbissenheit eines Kreationisten in einem Museum für Naturgeschichte «, ${ }^{31}$ meint etwa Philipp Blom über Holocaustleugner.

Kohärenzdominiertes Denken und Argumentieren »überzeugt Menschen, die sich auf ihre Erfahrungen nicht mehr verlassen wollen, weil sie sich mit ihnen in der Welt nicht mehr zurechtfinden können «. ${ }^{32}$ Die Neigung zu dieser die Wirklichkeit und Weltsicht strukturierenden Denkweise müsste demnach angesichts gestiegener Komplexität der

30 Vgl. Edward A. Shils, »Authoritarianism: >Right $<$ and >Left « «, in: Richard Christie / Marie Jahoda (Hg.), Studies in the Scope and Method of "The Authoritarian Personality ", Glencoe 1954, S. 24-49; Hans Jürgen Eysenck, The Psychology of Politics, London 1968; Milton Rokeach, The open and the closed mind, New York 1960.

31 Philipp Blom, »Copyright für ein Tabu« in: Cicero, Nr. 11 (2012), S. 21.

32 Arendt, Elemente und Ursprünge totaler Herrschaft. Antisemitismus, Imperialismus, totale Herrschaft, aaO. (FN 11), S. 966. 
Politik ${ }^{33}$ und bei gleich bleibender Ambiguitätssensibilität im späten 20. und frühen 21. Jahrhundert gewachsen sein: Weil Realitätserfahrungen häufig mit ihrem Weltbild, ihrer Erziehung und ihren Werten kollidieren, müssten Menschen nach Arendt dazu geneigt sein, sich bei der Beurteilung der Wirklichkeit und im Diskurs weniger auf die Empirie zu stützen, sondern auf ein Instrument, das Ordnung in das Chaos bringt, klare Entscheidungskriterien vorträgt, Verständnisstützen reicht und Widersprüchlichkeit negiert. Das Bedürfnis nach Klarheit, Struktur und Widerspruchsfreiheit (Vermeidung kognitiver Dissonanzen) findet Befriedigung in einem kohärenzdominierten Denkmuster, das sich in extremistischen Ideologien manifestiert.

Politischer Extremismus wird für Menschen mit einer niedrigen Ambiguitätstoleranz interessant, weil er in Form umfangreicher politischer Ideologien Aussagensysteme anbietet, die als Referenzrahmen für die Überprüfung des Wahrheitsgehaltes von empirischen Aussagen dienen können: etwa der Faschismus, der Salafismus, der Stalinismus. Dadurch gerät die empirische Wahrheit im Vergleich zur (ideo-)logischen Wahrheit ins Hintertreffen, ihre Bedeutung wird auf denjenigen Punkt reduziert, von dem aus logisch deduziert werden kann (Arendt).

Erst durch die logische Deduktion kann sich ein folgenreicher normativer Fehlschluss Bahn brechen: Die Realität wird der eigenen Gedankenwelt angepasst, sobald Extremisten Macht erlangen. Sie gehen dazu über, die Realität der eigenen Ideologie anzupassen. Hannah Arendt sprach hierbei vom »Ernstnehmen ideologischer Meinungen $\aleph^{34}$ und von der »Tyrannei des zwangsläufigen Schlußfolgerns « $:^{35} »$ Macht man damit ernst, daß im Kampf der Klassen es immer "absterbende« Klassen geben muss, so folgt daraus, daß man immer neue Gruppen der Gesellschaft ausrotten muss. Macht man damit ernst, daß es im Leben der Völker ebenso wie im Leben der Natur >Parasiten < gibt, so folgt daraus, daß man mit ihnen so umspringen darf wie mit Wanzen und Läusen, die man bekanntlich mit Giftgas ausrottet. « ${ }^{36}$

Das Hoffen einiger Menschen auf Kohärenz generierende Gesetzmäßigkeiten in der Politik wird mit dem dazu passenden Angebot seitens extremistischer Ideologien für den demokratischen Verfassungsstaat zu einem explosiven Gemisch. Daraus folgen jene Strukturmerkmale, die allen politischen Extremismen, unabhängig von ihrer Provenienz, eigen sind: exklusive Erkenntnis- und dogmatische Absolutheitsansprüche, essentialistische Deutungsmonopole, holistische Steuerungsabsichten, deterministische Geschichtsbilder, identitäre Gesellschaftskonzeptionen, dualistische Rigorismen und

33 Siehe exemplarisch Ulrich Beck, Risikogesellschaft. Auf dem Weg in eine andere Moderne, Berlin 1986; ders., Weltrisikogesellschaft. Auf der Suche nach der verlorenen Sicherheit, Berlin 2008.

34 Arendt, Elemente und Ursprünge totaler Herrschaft. Antisemitismus, Imperialismus, totale Herrschaft, aaO. (FN 11), S. 968.

35 Arendt, Elemente und Ursprünge totaler Herrschaft. Antisemitismus, Imperialismus, totale Herrschaft, aaO. (FN 11), S. 969.

36 Arendt, Elemente und Ursprünge totaler Herrschaft. Antisemitismus, Imperialismus, totale Herrschaft, aaO. (FN 11), S. 967-968. 
schließlich fundamentale Verwerfungen. Die Ablehnung der Offenheit des demokratischen Verfassungsstaates folgt daraus.

Die acht Strukturmerkmale extremistischer Doktrinen, die als Positivdefinition des Extremismus fungieren, lassen sich demnach auf die Prämisse von Extremisten zurückführen, die eigene, die Realität objektiv abbildende Ideologie sei logisch schlüssig aufgebaut. Diese Ideologie fungiert aus Sicht von Extremisten nicht als Theorie zur Erklärung einzelner politischer Zusammenhänge, die logisch und empirisch wahr sein sollte. Sie ist - so das implizite Axiom - ein Spiegelbild der Realität. Die Notwendigkeit des Nachweises empirischer Wahrheit muss daher Extremisten tautologisch erscheinen.

\section{Beispiele}

Ein erster Blick auf die Empirie soll erhellen, ob meine Behauptungen der Realität standhalten. Hierfür werden Beispiele - je aus dem Rechts- und Linksextremismus sowie aus dem Islamismus - vorgestellt.

Der Pazifismus des Linksextremismus etwa lässt den Gedanken aufkommen, Anarchisten, Autonome und Kommunisten lehnten jede Form der Gewaltanwendung ab. Der 1. Mai beweist indes jährlich das Gegenteil. Nun ist Militanz kein unumstrittenes Thema in der linksextremistischen Szene. Aufschlussreich ist es dennoch, zu hören, wie der Widerspruch zwischen Pazifismus und Steinewerfen aufgelöst werden kann. Während die Gewalt des Staates in Form von gesellschaftlichen Zwangsverhältnissen (Schule, Wehrdienst, Steuern, Gewaltmonopol des Staates) als strukturelle Gewalt und Repression aufgefasst wird und tätlicher Gewalt als ebenbürtig gilt, werden physische Angriffe etwa auf Polizisten nicht als Gewalt, sondern als Widerstand gegen den übermächtigen Staat gerechtfertigt. Von der linksextremen Szene ausgehende Gewalt wird allein durch die Neubezeichnung »Gegengewalt«, öfter noch: »Widerstand « umgedeutet. So kann der Widerspruch zwischen Einstellung (pazifistische Grundhaltung) und Handlung (Gewalt gegen den politischen Feind) aufgehoben werden, indem der Gewalt-Begriff umdefiniert wird.

In persönlichen Interviews stellt sich der Drang zu Kohärenz ähnlich dar: ${ }^{37}$ Gefragt nach seiner Herkunft, kommt ein interviewter Linksextremist auf seine Familie, die ihn vermutlich in einem linksalternativen Milieu sozialisiert hat, und seine Oma zu sprechen, die dem Nationalsozialismus angehangen habe. »[D]ie war dann ja eine von den Bösen«, so der Befragte, der hier offenkundig in zwei Farben malt. Dass seine Großmutter als Jugendliche dem Nationalsozialismus angehangen haben mag und sie heute dennoch eine liebenswürdige Person ist, wird nicht in Betracht gezogen. Dies schlägt sich in seinem

37 Ich danke dem Bundeskriminalamt für die Überlassung dreier anonymisierter Interviews und Saskia Lützinger für die zuvorkommende Kooperation im Rahmen der Interviewauswahl. Die Interviews stammen allesamt aus dem Projekt »Extremisten in biographischer Perspektive «, dessen Ergebnisse 2010 veröffentlicht wurden. Vgl. Saskia Lützinger, Die Sicht der Anderen. Eine qualitative Studie zu Biografien von Extremisten und Terroristen, Köln 2010. 
Bild von der Gesellschaft -»Alles sind dann [...] Spießer « - und vom Rechtsextremismus - »weil ich das einfach nicht sehen möchte« - nieder.

Ähnlich das Bild beim Islamismus: Wer ihm gegenüber argumentiert, die Demokratie bekämpfe nicht den Islam, sondern den Islamismus, und sei deshalb kein Feind, wird kaum auf offene Ohren stoßen. Vielmehr definiert sich der Feindstatus nicht aus dem, was die Repräsentanten der Demokratie tun, sondern aus dem, was eine autoritätshörige Interpretation des Islam gebietet. Keine noch so offene Gesellschaft wird einen Islamisten überzeugen, der etwa Sure 2, 191 zitiert: »Tötet sie [die Ungläubigen], wo immer ihr auf sie stoßt, und vertreibt sie, von wo sie euch vertrieben haben.«

Ein ähnlich ausgeprägter dualistischer Rigorismus findet sich bei der individuellen Argumentation: Opfer ist man [...] im Grunde genommen nur, wenn man Moslem ist « und »in erster Linie geht es immer [darum] den Feind zu begegnen, die neuen Kreuzritter, die Amerikaner [an anderer Stelle: »der Satan Amerika «] und die Israelis. Die muss man treffen und schlagen überall dann, wo man sie trifft. [... D]ie sind für das Unrecht und für den Zwist in der islamischen Gesellschaft verantwortlich.« Dazu zählen auch die »angeblichen Brüder, die in den normalen Moscheen verkehren«, also Muslime, die den Djihad ablehnen. Hier wird der politische Feind - nicht der Gegner - für Konflikte zwischen Staaten ebenso verantwortlich gemacht, wie innerhalb der Umma. Die eigene »Bruderschaft « wird wiederum positiv überhöht. Sie verdeutlicht die Bedeutung einer ideologisch kohärenten Umgebung: »[S]tetig haben wir die gleichen Gedanken und das gleiche Ziel und die gemeinsamen Ansichten.«

Darstellungen und Meinungen der »Mediengesellschaft « werden abqualifiziert, weil sie »Einfluss ausüben gegen uns. Gegen den Islam, gegen die Umma und gegen die Wahrheit«. Wahrheit wiederum bestehe im Kampf der Muslime gegen Nicht-Muslime. Vom eigenen Denksystem abweichende Behauptungen werden also prinzipiell negiert. Ebenfalls zu Tage tritt bei dem Befragten der exklusive Erkenntnisanspruch mit starker, weil religiöser Tendenz zum Antiempirismus: »[W]ir haben die Wahrheit erkannt, die Erleuchtung fanden wir im Wege Gottes. Der Islam ist die Lösung.« An anderer Stelle: »Ich kann nur allen Leuten, die ich treffe, raten, den wahren Pfad des Glaubens zu finden. Lest den Koran, lest die Botschaft, versteht sie«, fordert er und entlarvt das aus dem Islam abgeleitete essentialistische, dogmatisch vertretene Deutungsmonopol. Dass er alles, was außerhalb des Islam liegt, ablehnt, verurteilt und bekämpft, wird an anderer Stelle deutlich: »Alles was nicht muslimisch ist, muss man bekämpfen «, sagt er. Gleichzeitig offenbart er seinen intimen Wunsch nach einer einheitlichen Ideologie ohne Widersprüche, Nuancen oder Konflikte. Was außerhalb des Islam liegt, scheint für ihn eine existenzielle Bedrohung darzustellen: Der für ihn wichtige Djihad sei »Kampf um Gerechtigkeit, [...] gegen die Unterdrückung, [...] gegen die Hegemonie des Westens, $[\ldots]$ gegen schlechte Regierungen, $[\ldots]$ gegen Korruption, $[\ldots]$ gegen die ganzen Kriege, die vom Westen kommen, [...] für Identität, [...] für Freiheit, [...] für Kultur«. Ziel ist es, eine islamische, politisch homogene Weltgesellschaft zu ermöglichen.

Der Rechtsextremismus zeigt, wie erwähnt, ein ähnliches Denkmuster. So offenbart etwa die Leugnung des Holocaust allen Fakten zum Trotz ein kohärenzdominiertes Denkmuster. Ein interviewter Rechtsextremist ordnet seine Weltsicht ebenfalls nach ei- 
nem kohärenten Modell - dem Sozialdarwinismus: Er fordert, man solle »Behinderten das Lebensrecht entziehen, genau wie Homosexuellen«. Er geht erstaunlicherweise so weit, auch für sich dieses Prinzip konsequent anzuwenden: »[F]ür den Fall [...], dass ich an einem normalen Leben so gar nicht mehr teilhaben würde, $[\ldots]$ bin ich sofort einverstanden zu sagen, ok, dann weg damit.«

Die geordnete politische Weltsicht macht vor seinem Familienleben keinen Halt. Seinem Vater, der ihm gegenüber mehrfach derart gewalttätig gewesen sei, dass für den Befragten mehrwöchige Krankenhausaufenthalte folgten, wird an vielen Stellen des Interviews Lob und Dankbarkeit für die »deutsche Erziehung « zuteil. Was die Wahrnehmung internationaler politischer Prozesse angeht, so findet sich hier ebenfalls - in Form von antiamerikanischen und antisemitischen Verschwörungstheorien - kohärenzdominiertes Denken in seiner Reinform: Für alles Böse der Welt seien dunkle Kräfte im Hintergrund verantwortlich. Es befinde sich »alles in jüdischer Hand « bei einem großen »Hand-in-Hand-Spiel .

\section{Fazit}

Die acht Strukturmerkmale extremistischen Denkens lassen sich aus dem Versuch von Extremisten ableiten, Widersprüche in der Realität aufzulösen, indem sie als wahr anerkennen, was sich durch die von ihrer Ideologie postulierten Gesetzmäßigkeiten erklären lässt. Dadurch tritt eine unbedingte Dominanz des Kohärenzprinzips zutage, welches wiederum typisch extremistische Denkstrukturen zustande bringt. Empirische Aussagen sind demnach wahr, wenn sie widerspruchsfrei in ein umfassendes - die Realität vermeintlich objektiv abbildendes - Aussagensystem eingeordnet werden können.

Zugespitzt formuliert und auf idealtypische Demokratiefeinde bezogen, beruht ihr Denken auf dem »Pippi-Langstrumpf-Prinzip« (»Ich mach` mir die Welt, wie sie mir gefällt«), das politisches Lernen massiv erschwert. Es resultiert aus einem schwach ausgeprägten Vermögen, eigene Ansichten an der Realität zu adaptieren, sich also mit Widersprüchen, als unangenehm empfundenen Tatsachen und Konflikten auseinanderzusetzen und daraus in konstruktiver Weise Schlüsse zu ziehen. Stattdessen emanzipiert sich das politische Denken von der Realität und nimmt nur sporadisch Bezug auf jene Wirklichkeitsausschnitte, die das eigene Denken in logischen Bahnen stützen, um psychischen Stress zu vermeiden. Im demokratischen Spektrum wiederum überwiegt die Fähigkeit, als Wahrheitskriterium die Übereinstimmung von Aussage und Wirklichkeit auch dann anzuerkennen, wenn dies bedeutet, Konflikte und Zweideutigkeiten hinzunehmen und eigene politische Ansichten zu modifizieren, also zu lernen.

Nicht jeder, der in seinen politischen Ansichten um Widerspruchsfreiheit und Umfassendheit ringt, ist ein Extremist. Vielmehr gelten diese Prinzipien bei normativen Werte- und Überzeugungssystemen überall als erstrebenswert. Was das Denken von Extremisten ausmacht, ist die Anwendung eines logischen Wahrheitskriteriums auf Aussagen über die reale politische Realität und die damit verbundene Emanzipation von ihr. Wer auf die Übereinstimmung von Aussage und Realität als Kriterium der Wahrheit 
verzichtet, um die Widerspruchsfreiheit und die Universalität der eigenen Ideologie zu wahren, vermeidet Widersprüche zum Preis des Rückzugs aus der Realität.

Die Kontroverse zwischen Demokratiebefürwortung und -ablehnung ließe sich demnach ebenso gut auffassen als Konflikt zwischen Realitätsbezogenheit und -verschlossenheit. Er resultiert aus der (Un-)Fähigkeit, die Unübersichtlichkeit, Mehrdeutigkeit und Vielschichtigkeit der politischen Wirklichkeit zu akzeptieren. Während Freunde der offenen Gesellschaft nötigenfalls die Imperfektibilität und evolutionäre Entwicklung des Politischen anerkennen, leugnen die Feinde der offenen Gesellschaft diese Mängel der politischen Realität, indem sie von einer sich in sozialen Gesetzmäßigkeiten ausdrückenden, logischen Kohärenz der Politik ausgehen.

\section{Zusammenfassung}

Der Aufsatz versucht, das Phänomen des politischen Extremismus, der bisher ex negativo (Feindschaft gegenüber dem demokratischen Verfassungsstaat) und ex positivo (acht Strukturmerkmale extremistischen Denkens) definiert wurde, mithilfe eines sparsameren Beschreibungsmodells Herr zu werden. Der für Extremisten typische Doktrinrahmen lässt sich mithin aus dem Postulat ableiten, die politische Realität sei aufgrund sozialer Gesetzmäßigkeiten kohärent. Diesem Postulat ordnet sich die Beweisführung von Extremisten unter.

\section{Summary}

The study tries to define the phenomenon of political extremism with the help of a parsimonious frame. Whereas it seems clear that extremism can be described both with the help of its hostility towards constitutional democracy and with typical thinking patterns this approach tries to reduce the eight structural features of extremist thinking to one feature: the statement that political reality is coherent due to social laws.

Tom Mannewitz, »A really beautiful new world «-The coherence principle as a structural feature of political extremism 